See discussions, stats, and author profiles for this publication at: https://www.researchgate.net/publication/221639552

\title{
DVB Coverage Prediction Using Game Engine Based Ray-Tracing Techniques
}

Conference Paper in Vehicular Technology Conference, 1988, IEEE 38th · September 2011

DOI: 10.1109/NETECF.2011.6093238 · Source: DBLP

\section{CITATIONS}

10

4 authors:

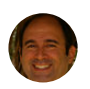

Andres Navarro

University ICESI

107 PUBLICATIONS 218 CITATIONS

SEE PROFILE

Narcís Cardona

Us: Universitat Politècnica de València

209 PUBLICATIONS 1,165 CITATIONS

SEE PROFILE

Some of the authors of this publication are also working on these related projects:

Security system to Android malware analysis View project

WiB - WideBand Reuse-1 System Concept for DTT View project
READS

73

Dinael Guevara

Universidad Francisco de Paula Santander

34 PUBLICATIONS 71 CITATIONS

SEE PROFILE

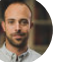

Jordi J. Gimenez

Institut für Rundfunktechnik

43 PUBLICATIONS 128 CITATIONS

SEE PROFILE 


\title{
DVB Coverage prediction using Game Engine based Ray-Tracing Techniques
}

\author{
Andres Navarro ${ }^{\#}$, Dinael Guevara ${ }^{*}$, Narcis. Cardona ${ }^{\alpha}$ and Jordi J. Gimenez ${ }^{\alpha}$ \\ ${ }^{\#}$ Universidad Icesi \\ Calle 18 No. 122-135, Cali, Colombia \\ anavarro@icesi.edu.co \\ *Universidad Francisco de Paula Santander \\ Circular 1 No.70-01, Medellin, Colombia \\ dinaelgi@ufps.edu.co \\ ${ }^{a}$ Universitat Politècnica de Valencia \\ Camino de Vera $S / N, 46022$ \\ ncardona@iteam.upv.es
}

\begin{abstract}
This paper presents the results of an evaluation of a propagation model based on ray-tracing techniques using a game engine and Graphics Processing Unit (GPU) in outdoor scenarios, as a gap-filler for a Digital Video Broadcasting Handheld (DVBH) service. This model involves complete identification of the parameters of wave propagation, multipath components between the transmitter and the receiver as attenuation, time delay of arrival (TDA), full polarimetric transmission matrix, direction of arrival DoA and the direction of departure DoD. In the paper, we present the results of the simulation and compare them with measurements, obtaining a satisfactory fit.
\end{abstract}

Index Terms-game engine, ray tracing, digital video broadcasting, wave propagation.

\section{INTRODUCTION}

$\mathrm{T}_{\mathrm{T}}^{\mathrm{he}}$ he European standard for mobile Digital Terrestrial Television (DTT), known as Digital Video BroadcastingHandhelds (DVB-H), is the technological evolution of the TDT standard, Digital Video Broadcasting-Terrestrial (DVBT) [1], adapted for mobile terminals. Its predominant feature is that it employs a discontinuous transmission technique in which information is transmitted in bursts, significantly reducing power consumption on the handset. Consequently low power transmitters or gap fillers can be used in of small or medium sized areas.

Multiple gap fillers need to be deployed to provide adequate levels of coverage. It is very common to use gap fillers as a complement to existing TDT networks, therefore the more severe conditions of DVB-T reception must be compensated for, especially for indoor and vehicular environments [2]. Gap fillers are feedback systems and many areas receive the signal from various locations. Distant interactions can generate excess delays, greater than the guard interval, thus generating interference, especially around nearby transmitters overhanging broad areas.

A typical TV network designed for indoor coverage or mobility requires a high number of low power gap fillers. Planning such a network using deterministic models, like ray tracing, requires a great deal of computational power and a lot of processing time, making these kinds of model undesirable for this purpose.

The use of empirical and statistical models are generalized to estimate path loss, because of their low complexity, high computational efficiency, sufficient accuracy and the need for only basic information about the environment. Recent research has analyzed and compared the performance of several radio propagation models (physical-statistical and empirical) for DVB-H networks with field measurement campaigns under different scenarios [3]. They found that these models are suitable for path loss estimation in DVB-H services, but the selection of the right propagation model has to be made depending on the deployment scenario, the available cartographic information, the transmitter height, and the terrain profile of the deployment scenario. Another significant conclusion is that measurement is necessary to adjust the parameters of propagation models. For estimated path loss, these types of model provide a good performance regarding processing time and precision.

Ray tracing [4] is one of the most used deterministic models for propagation prediction. Ray tracing is based on optics ray physics and allows precise characteristics of a radio signal in a complex environment to be obtained, such as attenuation, time delay, direction of arrival (DoA), and direction of departure (DoD).

There are few sources in the literature dealing with the use of ray-tracing techniques to obtain path loss and multi-path parameters for DVB-T services. Some research has documented the results of tests using those techniques in large environments and for DVB-T services, as well as research that analyzes the performance of using ray-tracing for the characterization of wideband in the context of DVB-T systems [5]. A 3D urban propagation model for large-scale and raytracing techniques was used. Measurement and simulation was carried out for a DVB-T transmitter located on the top of a hill in order to characterize and accurately estimate wide range 
echoes. The authors verified that ray-tracing is efficient for accurately estimating and characterizing high urban macrocells.

The use of ray-tracing techniques in medium size urban environments for mobile DVB-T services increases the computational time and the complexity. Some disadvantages of ray tracing, caused by the high density of buildings in an urban environment, complexity of buildings, number of considered effects needed to obtain good accuracy, number of rays launched and tested, and the many more severe conditions of reception, typical in mobile environments, makes the use of ray tracing techniques not particularly attractive as an alternative for DVB-T planning.

In this paper, we analyze the performance of a ray tracing model implemented using an Open Source Game Engine in an area of $2 \mathrm{~km} \times 2 \mathrm{~km}$ with a DVB-T transmitter located on the top of a building located in the campus of Valencia Technical University, and validates the results with drive test measurements along the Valencia Technical University campus and surroundings.

The propagation model used in this study is a ray-tracing model that predicts $3 \mathrm{D}$ contributions for urban environments using an open source game engine and GPU (Graphics Processing Unit) [6]. The idea is to verify the model proposed in different ambient and frequencies, validating the well known theory that ray tracing models work well for different frequencies. Also, we are testing our model in a quite bigger scenario than the reported in [6], reducing the processing time.

Game engines are powerful software packages that efficiently use data-structures and speed-up techniques to map 3D worlds with high-accuracy in real-time and with highprecision for physical simulations [7]. GPU based architectures provide a high memory bandwidth, and computational horsepower processing units. The ray tracing technique used is the "brute force" algorithm [8] or SBR (Shooting and Bouncing Rays) algorithm, which launches thousands, perhaps millions of rays in all possible directions of propagation from the transmitter. If a ray hits an object, then a reflecting ray and a refracting ray are generated. If a ray hits an edge, then a family of diffracting rays is generated, and each ray is traced to the next object and so on. Ray-tracing is currently the best solution to estimate site-specific space-time characteristics for narrowband or wideband urban propagation channels, which is essential for the simulation of the performance of new radio systems [9]. This radio propagation model is complemented with a detailed 3D urban model, gained from the transmitting and receiving antenna and their complex 3D directional pattern.

This paper is organized as follows: Section II describes the multipath propagation phenomena. Section III presents results from the measurements taken in the city of Valencia. The measurements are used to compare propagation model predictions with real data. Section III describes the predictions of the propagation channel used to obtain the $3 \mathrm{D}$ propagation model. Section IV presents the analysis and evaluation of the results. Finally, in section V we offer some conclusions.

\section{MULTIPATH PROPAGATION}

To estimate the parameters of multipath propagation due to reflections and diffractions is necessary to be able to distinguish between individual propagation paths. The SBR algorithm is particularly suitable for this task. The most important channel characteristics can be estimated by obtaining path parameters based on the frequency response $H(f, t)$ and the time-variant channel-impulse response of the channel $h(t, \tau)$.

The path parameters for the propagation between the transmitter and the receiver is defined by $n=1, \ldots, N(t)$ propagation paths [4]. The propagation parameters identified between the transmitter and the receiver are:

$\tau_{n}(t)$ : time delay of arrival (TDA) of path

$\overline{\mathrm{T}}_{n}(t)$ : full polarimetric transmission matrix of path

$\Omega_{\mathrm{T}, n}(t)$ : direction of departure (DoD) of path

$\Omega_{R, n}(t)$ : direction of arrival (DoA) of path.

At each interaction of the ray with an obstacle, the field strength is multiplied with a dyadic propagation transfer factor, which accounts for the actual propagation effect and for a change in divergence due to the interaction. Cascading all transfer factors (and therefore all occurring propagation phenomena) leads to the full polarimetric transmission matrix $\overline{\mathrm{T}}_{n}(t)$, which together with the path length (time delay $\tau_{n}(t)$ ) characterizes the field strength of the ray. $\Omega_{\mathrm{T}, n}(t)$ and $\Omega_{R, n}(t)$ are represented in colatitude and longitude (spherical coordinates) [9]. Also, we use the 3D gains of the transmitting and receiving antenna $G_{R}$ and $G_{T}$ and their complex directional pattern $\vec{C}_{R}$ and $\vec{C}_{T}$.

The frequency response of the channel is:

$$
\begin{aligned}
H(f, t)= & \sqrt{\left(\frac{C_{O}}{4 \pi f_{C}}\right)^{2} G_{R} G_{T}} \cdot \sum_{n=1}^{N(t)} \vec{C}_{R}\left(\Omega_{R, n}(t)\right) \\
& \cdot \bar{T}(t) \vec{C}_{T}\left(\Omega_{T, n}(t)\right) e^{-j 2 \pi f \tau_{n}(t)} \\
= & \sum_{n=1}^{N(t)} A_{n}(t) e^{-j 2 \pi f \tau_{n}(t)}
\end{aligned}
$$

Where $C_{O}$ is the speed of light in a vacuum and $f_{\mathcal{C}}$ is the center frequency of the system. $A_{n}(t)$ represents the complex amplitude of the $n^{\text {th }}$ multipath component and incorporates the properties of the transmitter and receiver antenna [10].

The low-pass impulse response of the channel $h(t, \tau)$ is obtained by the inverse Fourier transform of (1).

$$
h(\tau, t)=\sum_{n=1}^{N(t)} A_{n}(t) e^{-j 2 \pi \tau_{c} \tau_{n}(t)} \delta\left(\tau-\tau_{n}(t)\right)
$$

Thus, the channel model could be represented as a power delay profile (PDP) expressed by (2). 
We have used ray-tracing in order to obtain the parameters of the multipath propagation channel, and so are able to estimate path loss.

\section{MEASUREMENT RESULTS}

This section reports the measurement campaign for characterization of the propagation model around a small macrocell sub-urban transmitter in the city of Valencia. That measurement campaign was carried out by the iTEAM research institute inside and out of the main campus of the Universidad Politécnica de Valencia.

Figure 1 shows the transmitter, which is located on the top of building inside of the main campus, $24 \mathrm{~m}$ in height, thus providing medium coverage; the EIRP of all the transmitters is $36.01 \mathrm{dBm}$. The transmitter has two panel antennas oriented at $114^{\circ}$ and $316^{\circ}$ azimuth. The antennas are a verticallypolarized panel type with $62^{\circ}$ half-power beam-width in the horizontal plane, $28^{\circ}$ in the vertical plane and $12.15 \mathrm{dBm}$ gain.

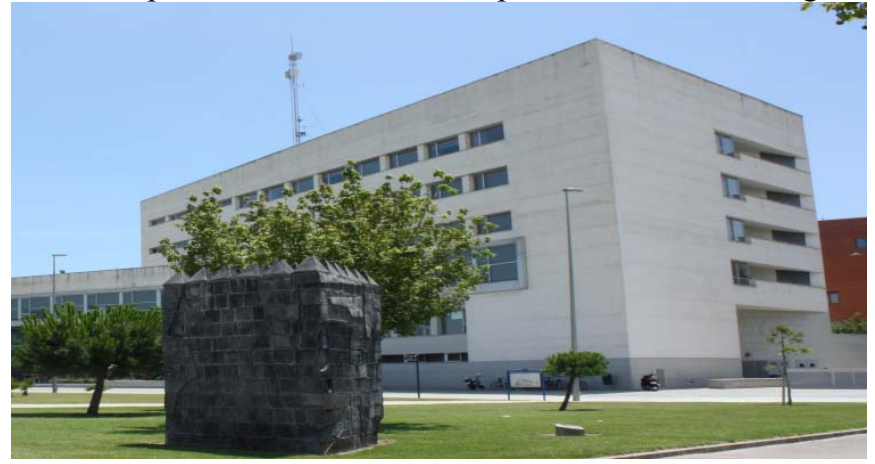

Figure 1 Site location of the transmitter on the top of the building inside of the main campus.

Figure 2 shows the measurement scenario. This scenario is $2 \mathrm{~km} \times 2 \mathrm{~km}$ in area, with slowly inclined streets and a complex of buildings around the campus.

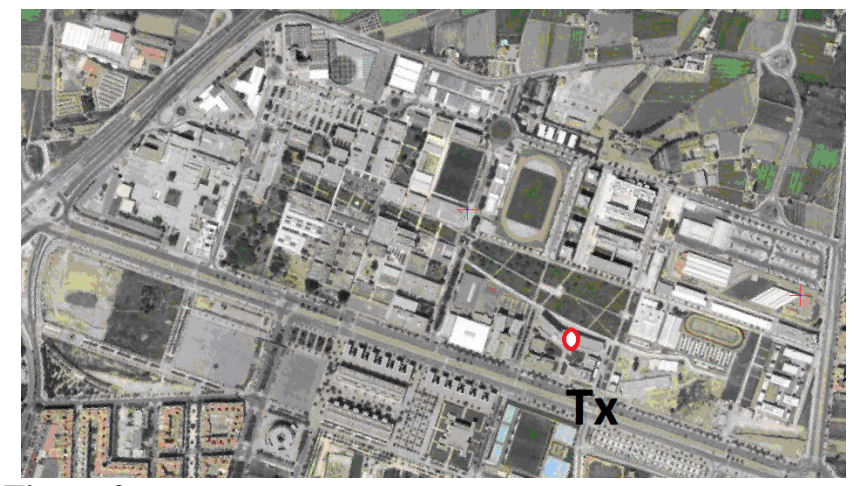

Figure 2 Main campus of Valencia Technical University, the red point indicates the transmitter location.

The measurement campaign consists in collecting the received power in the streets, inside and out the main campus and around the transmitter. Figure 3 shows the different receiver points. It is localized centered along to the street.

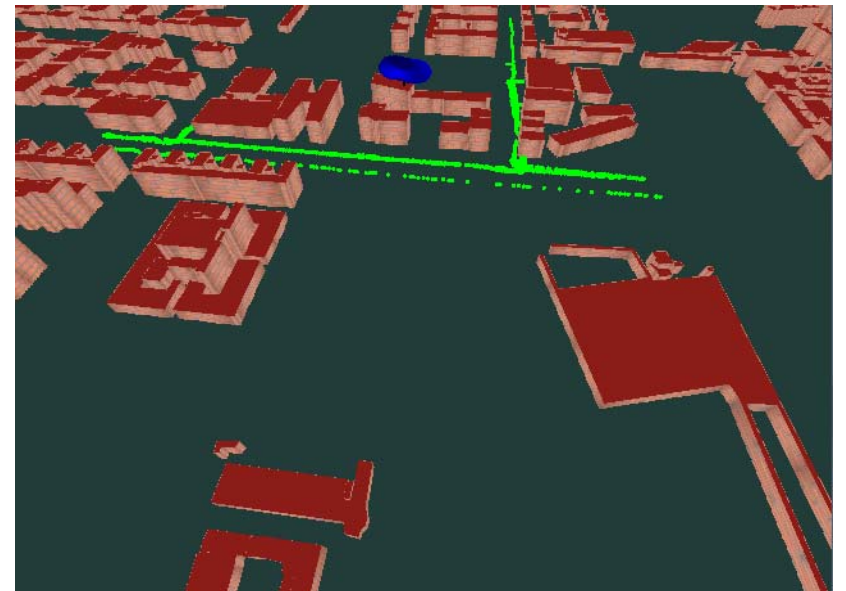

Figure 3 Measurement points location (green spheres) for LOS and NLOS. Transmitter localization is indicated with a blue bounding

The transmitter provided a DVB signal on $496 \mathrm{MHz}$. The receiver system measures the power received within the whole signal bandwidth, i.e. approximately $8 \mathrm{MHz}$. The receiver system consist of Teamcast professional receiver, a GPS and a software-based measurement system developed by iTEAM. The acquisition time was $1 \mathrm{~ms}$. The receiving antenna consists of a vertical quarter-wave length monopole positioned above a metallic plate fixed on the top of a moving vehicle. Total power provided to the transmitter antenna is monitored throughout the measurement campaign to control the stability of the transmission. The measurement permits the characterization of the propagation loss to evaluate the performance of the propagation model for DVB-T as well as DVB-H services for the mobile terminal. The selected routes for all the measurements include localizations with LOS and NLOS. We obtained 1380 measurement points for the drive test.

\section{PREDICTION OF THE PROPAGATION CHANNEL}

One of the necessary conditions in order to obtain reliable results when using a $3 \mathrm{D}$ ray tracing model is the need for a $3 \mathrm{D}$ urban model with excellent resolution and detail. In order to obtain such a 3D model, we used a $1 \mathrm{~m}$ resolution ASCII raster DTM of the city of Valencia. This is a complex environment with many buildings, irregular roofs and different structures, requiring that the processing speed and the data-structure be used efficiently. This DTM was converted in a 3D model using spatial geometries representing streets, roofs and walls. Figure 4 shows the 3D model implemented, where we used flat polygons to represent external faces of geometries, because of their simplicity and ease of automating the process from the raster format, cylinders represent edges (not visible in the figure) and spheres represent reception points, using the format required by the Game Engine. 


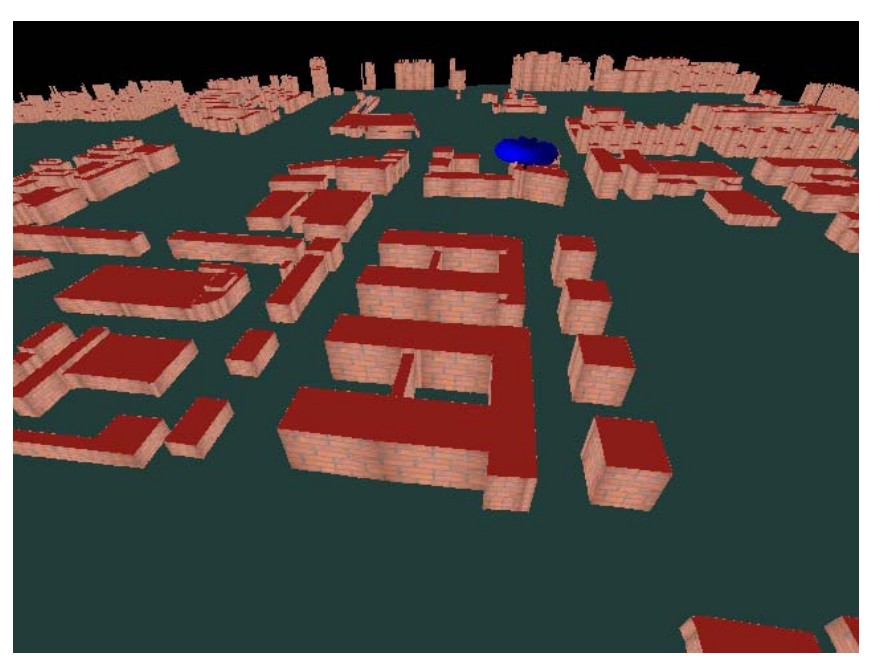

Figure 4 3D Model for scenario around of transmitter using the game engine

We store and manipulate constitutive parameter information as an attribute within our 3D Model (i.e. permittivity, permeability and rugosity). According to their electromagnetic properties, the structure of the buildings and streets are classified into 3 different classes with common dielectric material parameters for building walls, building roofs and street pavement. We assumed that the building walls and objects in the selected area of the city have the same constitutive parameters (i.e. permittivity and permeability) and assume brick ( $\varepsilon r=7-j 0.3, \mu r=1)$ as the building material, for street pavement and building roofs we assume dry concrete $(\varepsilon r=5.3-\mathrm{j} 0.25, \mu \mathrm{r}=1)$. Finally we assume that brick and dry concrete have a similar rugosity factor $(\sigma \mathrm{r}=1 \mathrm{~mm}$. $)$ [11].

The number of iterations is limited to five combinations in total. The number of diffractions is limited to two and the number of reflections considered is five. Therefore, that number of interactions increases the computational complexity and processing time. To obtain sufficient accuracy with respect to measurements, experience has shown that a number of five wave interactions (reflection, diffraction) are sufficient. Several references indicate similar results [12].

The icosahedron used for ray launching has a tessellation frequency of 256 for high precision. A high tessellation reduces the maximum radio of the reception sphere, so therefore incrementing the detection probability, the mean angular separation we use between neighboring rays in the $3 \mathrm{D}$ space is $0.27^{\circ}$ (i.e. 655362 launched rays) which increases the computational complexity.

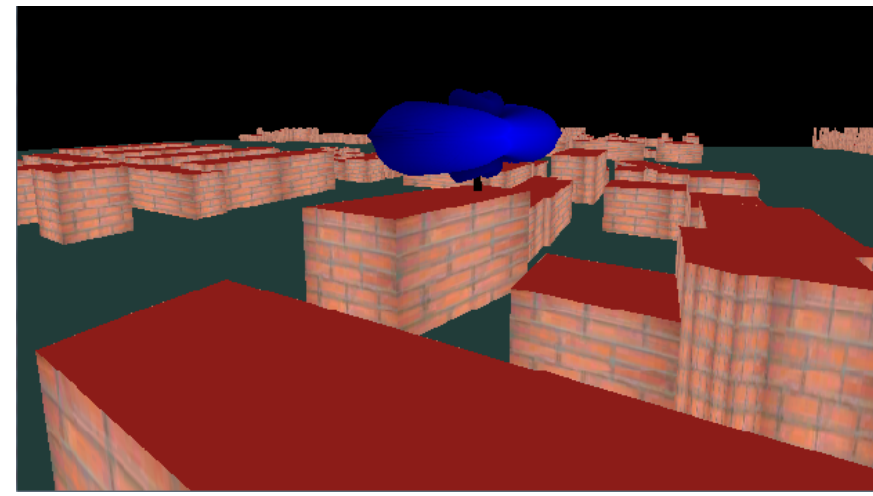

Figure 5 3D Model for transmitter antenna. This is an augmented version for visualization effects.

We have modeled both the transmitter and receiver antennas in 3D using their complex 3D directional pattern. Figure 5 shows the $3 \mathrm{D}$ model of the directional pattern obtained from 2D vertical and horizontal directional patterns, using the interpolation method to obtain the $3 \mathrm{D}$ patterns as described in [13].

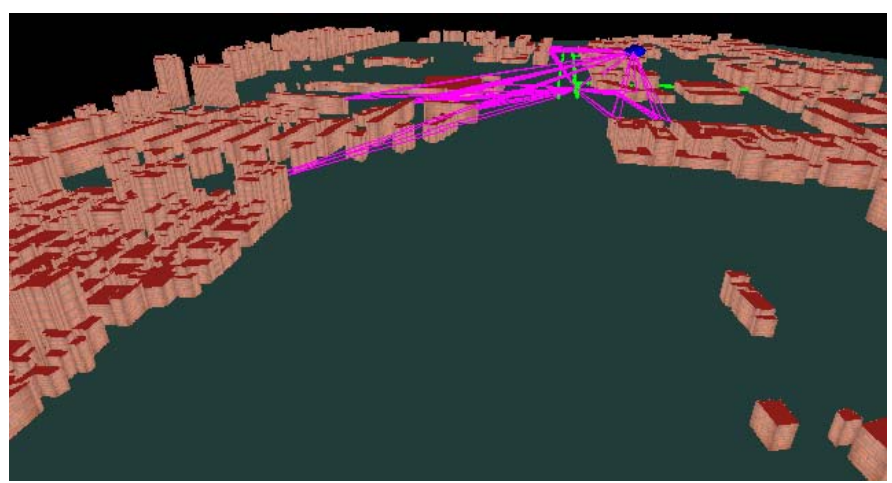

Figure 6 Reflected and diffracted rays for a reception point. The ray tracing used the game engine and card graphics.

Figure 6 shows some of the results of a ray tracing procedure, illustrating reflections and diffractions. The raytracing procedure estimates the propagation parameters using the game engine. The parameters obtained are TDA, full polarimetric transmission matrix, DoD and DoA. These parameter values used to simulate path loss are also useful to investigate the narrowband, wideband and spatial characteristics of the propagation channel.

In addition, we calculated the local mean of the absolute value of the simulated path loss curves using a sliding window of $40 \lambda$ in length [14].

For the simulation process, we have used a desktop computer with an Intel Core i7-940 processor with $2.93 \mathrm{GHz}$ clock speed, and 6GB RAM; equipped with a SLi, Dual NVIDIA GeForce GTX280 Graphics accelerator with 240 flow processors and 1024MB Video RAM with hardware integrated physics analysis.

\section{ANALYSIS AND RESULTS}

It is well known that ray tracing models can predict not only path loss but also channel parameters as our model does. 
However, for this paper we analyzed the model's precision with regards to path loss only. For this analysis we use statistical parameters like standard deviation, mean absolute error and mean error, and the correlation coefficient.

Fig. 4 shows the scenario used for the simulations. It consisted of 15747 bounding polygons to walls, 1809 bounding polygons to roofs, 20 bounding polygons to streets, 10291 bounding cylinders to edges horizontal, 9042 bounding cylinders to edges verticals and 1380 bounding spheres to reception points. The processing time was few seconds.

We obtained the propagation path parameters values using ray tracing with the game engine and GPU. The parameters obtained for power prediction are TDA, full polarimetric matrix, DoD and DoA. These values, that were used for the estimation of the propagation model and power prediction, were also used to estimate the power delay profile at each reception point.

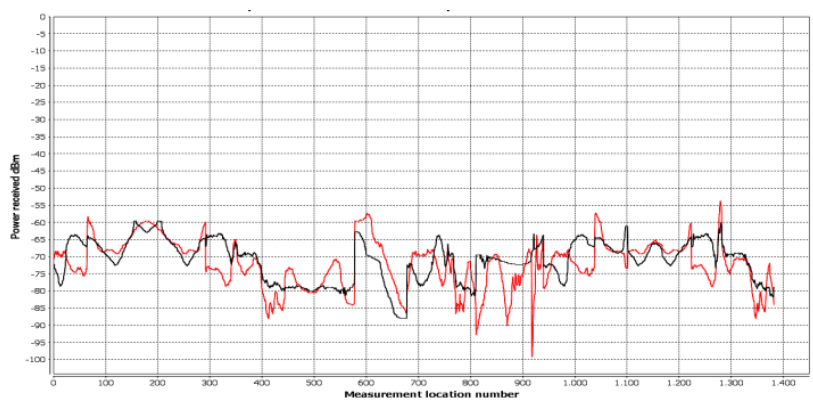

Fig 6. Power prediction comparison of the ray tracing output (red line) with the measurements (black line).

The processing time was 384 mins for all the results for the propagation model. This time includes the analysis for 1380 reception points and 120'470.065 processed rays, therefore this requires high performance in computational time to process the propagation path parameters in areas of medium size, with high complexity and large numbers of reception points, as in a $3 \mathrm{D}$ propagation model for medium-scale to DVB services for mobile terminals. Computational time of ray tracing using the game engine and GPU, is more sensitive to the size of the area of interest and complex environment, but is less sensitive to large numbers of reception points.

Fig. 6 shows the power prediction comparison of the raytracing output (red line) with the measurements (black line). This comparison shows a high accuracy between the estimated powers and the measured ones in some sections of the measurement route (i.e. location numbers from 75 to 300,580 to 680 , etc.). Table I summarizes the statistical results for path loss prediction. Predicted results fit well with the measurement data, with an absolute mean error of $4.64 \mathrm{~dB}$, mean error of $1.05 \mathrm{~dB}$, standard deviation of $6.89 \mathrm{~dB}$ and a correlation coefficient of 0.58 . The value of the mean error is within the margin of 4 to $8 \mathrm{~dB}$ of predictions for very high accuracy as compared with [15], [16] and [17].
TABLE I. STATISTICAL SUMMARY OF PATH LOSS PREDICTION.

\begin{tabular}{|c|c|c|c|}
\hline $\begin{array}{c}\text { Mean } \\
\text { absolute } \\
\text { error } \\
\text { (dB) }\end{array}$ & $\begin{array}{c}\text { Mean error } \\
\text { (dB) }\end{array}$ & $\begin{array}{c}\text { Standard } \\
\text { Deviation } \\
\text { (dB) }\end{array}$ & Correlation \\
\hline 4.64 & -1.05 & 6.89 & 0.58 \\
\hline
\end{tabular}

The results of the error statistics for ray-tracing have better accuracy than some propagation non deterministic models. We have compared our results with Okumura-Hata, Xia-Bertoni and Hata-Deygout, obtained from the research for DVB-H networks with field measurement campaigns in different scenarios as reported in [3].

\section{CONCLUSIONS}

We have presented the results for estimated propagation path loss parameters to DVB services for mobile terminals using ray tracing techniques with a Game Engine and GPU. This method shows a high performance in $3 \mathrm{D}$ complex environment models and a high-accuracy. The results obtained from the comparison between the predictions and measurements shows a good accuracy for the proposed model.

The proposed model also performs well with regards to drive tests in areas of medium size, high complexity and with a large number of reception points, as in a $3 \mathrm{D}$ propagation model for medium-scale DVB services for mobile terminals.

\section{REFERENCES}

[1] G. Faria, J. A. Henriksson, E. Stare, and P. Talmola, "DVB-H: Digital Broadcast Services to Handheld Devices," Proc. IEEE, vol. 94, no. 1, pp. 194-209, Jan. 2006.

[2] D. Gómez-Barquero and A. Bria, "Feasibility of DVB-H Deployment on Existing Wireless Infrastructure," Proc. International Workshop on Convergent Technologies (IWCT), Oulu, Finland, 2005

[3] A. Salieto, G. Roig, D. Gómez-Barquero and N. Cardona, "Radio Propagation Models for DVB-H Networks," Proc. of the Fourth European Conference (EUCAP), Barcelona, Spain, 2010.

[4] C. Balanis, Advanced Engineering Electromagnetics. New York: Wiley, 1999.

[5] Y. Corre and Y. Lostanlen, "Characterization of the Wideband Wireless Channel in the Context of DVB Systems," IEEE 19th International Symposium (PIMRC), Cannes, France, 2008.

[6] A. Navarro and D. Guevara, "Applicability of Game Engine for Ray Tracing Techniques in a complex urban environment", IEEE 72nd Vehicular Technology Conference - VTC-Fall 2010, pp. 1-6, Sept. 2010.

[7] P. McDowell, R. Darken, J. Sullivan and E. Johnson, "Delta3D: A Complete Open Source Game and Simulation Engine for Building Military Training Systems", JDMS, vol. 3, no. 3, 2006.

[8] U. Dersch and E. ZJollinger, "Propagation Mechanism in Microcell and Indoor Environments," IEEE Transactions on Vehicular Technology, VT-43,4, pp. 1058-1066, Nov. 1994.

[9] L. M. Correia, Mobile Broadband multimedia networks. Report on the research work of COST 273. Academic Press, 2006.

[10] T. Fugen, J. Maurer, T. Kaiser and W. Wiesbeck, "Capability of 3-D Ray Tracing for Defining Parameter Sets for the Specification of Future Mobile Communications Systems," IEEE Transactions on Antennas and Propagation, vol. 54, pp. 3125-3137, 2006.

[11] S. Stavrou and S. R. Saunders, "Review of constitutive parameters of building materials," Proc. 12th Int. Con.f Antennas and Propagation (ICAP '03), vol. 1, pp. 211-215, Exeter, UK, Mar.-Apr. 2003. 
[12] K. A. Chamberlin and R. J. Luebbers, "An Evaluation of Longley-Rice and GTD Propagation Models," Transactions on Antennas and Propagation, AP-30, vol. 30, pp. 1093-1098, Nov. 1982.

[13] F. Gil, A. R. Claro, J. M. Ferreira, C. Pardelinha and L. M. Correia, "A 3D interpolation method for base-station-antenna radiation patterns," IEEE Antennas and Propagation Magazine, vol. 43, no. 2, pp. 132-137, Apr. 2001.

[14] W. C. Y. Lee, Mobile Communications Engineering. New York: McGraw-Hill, 1982.

[15] K. R. Schaubach, N. J. D. IV, and T. S. Rappaport, "A ray tracing method for predicting path loss and delay spread in microcellular environments," Proc. IEEE Vehicular Technology Conference, vol. 2, pp. 932-935, May 1992.

[16] A. Schmitz and L. Kobbelt, "Wave propagation using the photon path map," PE-WASUN '06, New York, pp. 158-161, 2006.

[17] S.-C. Kim, B. J. Guarino, T. M. W. III, V. Erceg, S. J. Fortune, R. A. Valenzuela, L. W. Thomas, J. Ling, and J. D. Moore, "Radio propagation measurements and prediction using three-dimensional ray tracing in urban environments at $908 \mathrm{Mhz}$ and $1.9 \mathrm{Ghz}$," IEEE Transactions on Vehicular Technology, vol. 48, pp. 931-946, 1999. 